\title{
Integración de la gestión del riesgo para el cumplimiento de altos estándares de calidad en el sector salud en Colombia*
}

\section{Risk management integration for the compliance of high quality standards in the health sector in Colombia}

Recibido: 11 de febrero de 2014 Revisado: 14 de abril de 2014 Aceptado: 27de junio de 2014

Gloria Milena Vanegas Rodrígue****

E.S.E Salud Sogamoso

\section{RESUMEN}

Las Instituciones Prestadoras de Servicios de Salud (IPS) en Colombia están reglamentadas por el Sistema Obligatorio de Garantía de la Calidad (SOGC). El componente de Acreditación en Salud de este sistema contempla que se adelanten acciones para gestionar los riesgos asistenciales de las IPS, pero también que se integre el manejo de los riesgos derivados de los procesos administrativos; para gestionar estos últimos, las empresas del sector público deben cumplir con lo establecido por el Modelo Estándar de Control Interno (MECI) y la Norma Técnica de Calidad de la Gestión Pública (NTCGP 1000), con el fin que las Instituciones mejoren continuamente su desempeño y realicen un control razonable de su gestión.

Esta investigación propone una herramienta de gestión o matriz de control, que a través del enfoque de un sistema de gestión del riesgo, contribuye a: identificar y valorar los riesgos (positivos y negativos), establecer un plan de acción por áreas de la organización,

* Artículo de investigación.

* Gloria Milena Vanegas Rodríguez. Especialista en Gerencia de Instituciones de Salud; Magister en calidad y gestión integral de la Universidad Santo Tomás. Correo electrónico: Vanegas.gloria@yahoo.es 
controlar el seguimiento y evaluación de las acciones encaminadas a cumplir altos estándares de calidad, lo cual propicia la optimización de recursos en la IPS, identifica riesgos antes invisibles y facilita la implementación de los diferentes estándares. La recolección de la información para poner a prueba la hipótesis de investigación se realizó mediante encuestas, análisis de documentación y revisión de referencias bibliográficas relacionadas con el sistema obligatorio de garantía de la calidad en salud y la gestión del riesgo según se establece en la norma técnica ISO 31000:2009.

Palabras clave: gestión del riesgo, integración de sistemas de gestión, ISO 31000, Sistema Obligatorio de Garantía de la Calidad en Salud.

\section{ABSTRACT}

The health service institutions in Colombia (IPS) are regulated by the Obligatory System of Health Quality Assurance (SOGC). The Health Accreditation component of this system provides that actions be brought forward to manage health risks of IPS but also that integrates the management of risks arising from administrative processes. To manage the latter, public sector organizations must comply with the provisions of the Standard Model of Internal Control (MECI) and the Technical Standard for Quality of Public Management (NTCGP 1000), so that the institutions continuously improve their performance and make a reasonable management control. This research proposes a management tool or matrix control, through a system approach to risk management helps to: identify and assess the risks (positive and negative), establish an action plan for areas of the organization, control monitoring and evaluation of actions to meet high quality standards, which leads to the optimization of resources in the IPS, identifies risks before unseen and facilitates the implementation of the different standards. Gathering information to test the research's hypothesis was conducted through surveys, analysis documentation and review of references related to the Obligatory System of Health Quality Assurance and risk management as established in the technical standard ISO 31000:2009.

Keywords: Risk management, management systems integration, ISO 31000, Obligatory System of Health Quality Assurance.

\section{INTRODUCCIÓN}

Las Instituciones Prestadoras de Servicios de Salud (IPS) se encuentran obligadas a cumplir leyes y normas relacionadas con la gestión de la calidad, tendientes a que sus procesos contribuyan a mejorar la calidad en la prestación de los servicios de salud; el cumplimiento de dichos propósitos comúnmente se ve afectado por elementos internos atribuibles a factores que aumentan el riesgo en la gestión de las organizaciones (Chomalí y Miranda, 2003). Las instituciones son afectadas en su gestión por la incertidumbre y el principal reto para la gerencia es determinar cuánta incertidumbre acepta para equilibrar sus objetivos estratégicos; para ello debe gestionar los riesgos. Según Devi Puerto (2011), el concepto de sistema de gestión del riesgo se define como un proceso sistemático que permite la mejora continua en la toma de decisiones y en el desempeño de las organizaciones, en aras de lograr los objetivos establecidos, mediante la determinación, la evaluación el análisis y el tratamiento de los riesgos; como modelos de gestión que incorporan el análisis de riesgos, se resaltan la norma técnica NTCGP 1000:2009 (ICONTEC, 2009b), el MECI:2005 (Departamento Administrativo de la Función Pública, 2005) y el Manual de Acreditación 
en Salud Ambulatorio y Hospitalario (Ministerio de la Protección Social, 2011); la norma técnica NTC ISO 31000:2009 (ICONTEC, 2009a) trata sobre la gestión integral del riesgo.

Para el desarrollo de esta investigación se definieron cuatro hipótesis que establecen relación de causalidad, descritas así: 1. La gestión del riesgo contribuye a cumplir los objetivos estratégicos de la IPS; 2. Durante el proceso de atención al usuario en la IPS, no se identifican la totalidad de los riesgos, en consecuencia no se adoptan mecanismos de gestión de tales riesgos; 3 . El tratamiento de los riesgos de una institución de salud favorece la mitigación de dichos riesgos; 4. La articulación de la gestión del riesgo y las evaluaciones internas de calidad favorecen el cumplimiento de los estándares de calidad en la atención en salud.

Durante el proceso de la investigación se puso a prueba la segunda hipótesis dando como resultado que durante el proceso de atención al usuario en la IPS, no se identifican la totalidad de los riesgos, por ende no se adoptan mecanismos de prevención y gestión de tales riesgos, resultando afirmativa la hipótesis inicialmente descrita; de igual manera, gracias a la matriz de correspondencia se logra probar la cuarta hipótesis, analizando que sí es posible articular la gestión del riesgo y las evaluaciones internas de calidad, para contribuir al cumplimiento de los estándares de calidad, del proceso de atención al cliente asistencial.

El desarrollo de la investigación permitió desarrollar una propuesta de aplicación de la gestión del riesgo en la Empresa Social del Estado (E.S.E) Salud Sogamoso, que sirvió para apoyar el cumplimiento de los estándares del proceso de atención al cliente asistencial según el Manual de acreditación en salud ambulatorio y hospitalario.

\section{METODOLOGÍA}

El fundamento epistemológico (López, 2011) del trabajo de investigación se tomó a partir de la teoría de Reason (2000), definida en el Modelo del "queso suizo" (Figura 1), la cual afirma que el error aparece cuando el proceso de atención no se ha diseñado con un enfoque sistémico, en consecuencia, conforme a Astolfo Franco (2005), cuando un error ocurre es el resultado de una secuencia de procesos defectuosos que han favorecido la aparición del error o no lo han prevenido; según este modelo, los controles que establece una organización para impedir la aparición de fallas se pueden representar como rebanadas de queso; los agujeros en esas rebanadas son los puntos débiles del sistema. Cuando por alguna razón se alinean los agujeros, se materializa un riesgo y se presenta una falla, accidente 0 evento adverso.

Figura 1. Modelo del queso suizo

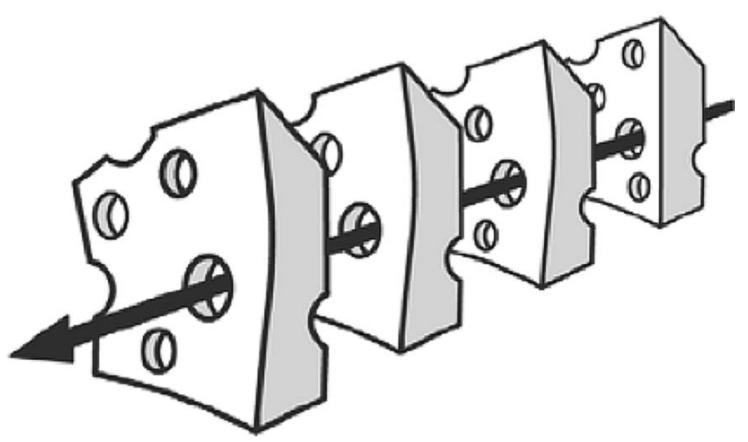

Fuente: Reason, 2000.

Se realiza una revisión literaria de los tipos de riesgos presentes en las organizaciones (Departamento Administrativo de la Función Pública, 2011; Urrego, 2008; Espinal, Yepes y Garcia, 2010); para el propósito de esta investigación se consideran ocho riesgos principales: estratégico, de imagen, operativo, financiero, de cumplimiento, de tecnología, ocupacional y asistencial. 
Conforme a la información brindada por Hernández, Fernández y Baptista (1997), para la recolección de información, se elabora el siguiente plan teniendo en cuenta las variables, la muestra y los recursos disponibles (Tabla 1.).

Se utiliza como fuente de información real la E.S.E Salud Sogamoso; inicialmente, se procede a revisar información del documento interno "Componente Administración del Riesgo" - Elemento estratégico del MECI, y del documento planeación estratégica sistema de gestión de la calidad. Esta revisión documental permitió determinar los elementos estratégicos que se utilizan como parte integrante de la gestión del riesgo a saber:

a. Diagnóstico de las variables en términos de fortalezas y debilidades

b. Formulación de estrategias

c. Matriz de riesgo por procesos de la IPS

d. Formatos de registro de riesgo e. Programa y plan de tratamiento de riesgos

f. Plan de acción de riesgos

g. Identificación del riesgo

El análisis de la revisión documental, contribuyó con el desarrollo de la investigación en los siguientes aspectos:

- Elaboración de las preguntas de la encuesta.

- Aporte en los elementos estructurales de propuesta de aplicación de la gestión del riesgo desde la norma técnica NTC ISO 31000, como apoyo al cumplimiento de los estándares del proceso de atención al cliente asistencial del sistema único de acreditación; específicamente los siguientes: Integración de las acciones de la gestión del riesgo y el sistema de gestión de la calidad; promoción del trabajo en equipo; descripción de mecanismos de comunicación de las acciones realizadas; optimización y unificación de formatos establecidos.

Tabla 1.Plan de recolección de datos

\begin{tabular}{|l|l|l|l|}
\hline \multicolumn{1}{|c|}{ Fuentes de información } & \multicolumn{1}{|c|}{$\begin{array}{c}\text { Localización } \\
\text { de las fuentes }\end{array}$} & \multicolumn{1}{|c|}{ Método para recolectar los datos } & \multicolumn{1}{c|}{$\begin{array}{c}\text { Método para el análisis } \\
\text { de los datos }\end{array}$} \\
\hline $\begin{array}{l}\text { Líderes de procesos de } \\
\text { la IPS. }\end{array}$ & $\begin{array}{l}\text { Instalaciones de } \\
\text { la IPS. }\end{array}$ & Encuesta. & $\begin{array}{l}\text { Tabulación de datos en tabla de } \\
\text { Excel y presentación en gráficos. }\end{array}$ \\
\hline $\begin{array}{l}\text { Documentos de gestión } \\
\text { de la IPS. }\end{array}$ & $\begin{array}{l}\text { Instalaciones de } \\
\text { la IPS, oficina de } \\
\text { Control Interno. }\end{array}$ & $\begin{array}{l}\text { Solicitud y revisión del documento interno } \\
\text { "Componente Administración del Riesgo" - } \\
\text { Elemento estratégico del MECI, y del documento } \\
\text { planeación estratégica sistema de gestión de la } \\
\text { calidad. }\end{array}$ & $\begin{array}{l}\text { Comparación de datos con la } \\
\text { tabulación de encuestas y las } \\
\text { directrices de la NTC ISO } \\
\text { 31000. }\end{array}$ \\
\hline $\begin{array}{l}\text { Normas para la gestión } \\
\text { del riesgo y manual } \\
\text { hospitalario y ambulatorio } \\
\text { de acreditación en salud. }\end{array}$ & $\begin{array}{l}\text { Documento trabajo } \\
\text { de grado y redes de } \\
\text { internet. }\end{array}$ & $\begin{array}{l}\text { Revisión del documento interno "Componente } \\
\text { Administración del Riesgo" - Elemento estratégico } \\
\text { del MECI, y del documento planeación estratégica } \\
\text { sistema de gestión de la calidad. }\end{array}$ & $\begin{array}{l}\text { Matriz de correspondencia } \\
\text { entre las normas y el Manual de } \\
\text { Acreditación en Salud. }\end{array}$ \\
\hline $\begin{array}{l}\text { Líderes de procesos } \\
\text { Institución prueba piloto. }\end{array}$ & $\begin{array}{l}\text { Instalaciones de la } \\
\text { piloto. }\end{array}$ & $\begin{array}{l}\text { Prueba piloto del Instrumento. } \\
\text { Compruebaración de las respuestas }\end{array}$ \\
\hline
\end{tabular}

Fuente: elaboración propia. 
Se elabora una matriz de correspondencia para identificar las convergencias y complementariedades de los modelos que se consideraron; la Tabla 2 ejemplifica los aspectos afines entre la Norma Técnica de Calidad en la Gestión Pública NTCGP1000:2004, el modelo estándar de control interno MECI:2005, la NTC ISO 31000:2009 y el Manual de acreditación en salud ambulatorio y hospitalario.

Tabla. 2. Matriz de Correspondencia grupo de estándares del procesos de atención al cliente asistencial

\begin{tabular}{|c|c|c|c|c|c|c|c|c|c|}
\hline \multicolumn{2}{|c|}{ NTCGP 1000:2009 } & \multicolumn{3}{|c|}{ MECI:2005 } & \multicolumn{2}{|c|}{ NTC ISO 31000} & \multicolumn{2}{|c|}{$\begin{array}{c}\text { Manual de acreditación } \\
\text { en salud ambulatorio y } \\
\text { hospitalario }\end{array}$} & \multirow[t]{2}{*}{ Riesgos } \\
\hline Titulo & $\mathbf{N}$ & Subsistema & Componente & Elementos & Titulo & $\mathbf{N}$ & Titulo & $\mathrm{N}$ & \\
\hline \multirow[t]{2}{*}{$\begin{array}{l}\text { Gestión } \\
\text { documental }\end{array}$} & \multirow[b]{2}{*}{4.2} & \multirow{2}{*}{$\begin{array}{l}\text { Control de } \\
\text { gestión }\end{array}$} & \multirow{2}{*}{$\begin{array}{l}\text { Actividades } \\
\text { de control } \\
\text { Información }\end{array}$} & & \multirow{2}{*}{$\begin{array}{l}\text { Registro de los } \\
\text { procesos para } \\
\text { la Gestión del } \\
\text { Riesgo }\end{array}$} & \multirow[b]{2}{*}{5.7} & \multirow{2}{*}{$\begin{array}{l}\text { Grupo de } \\
\text { estándares de } \\
\text { gerencia de la } \\
\text { Información }\end{array}$} & \multirow[b]{2}{*}{7.7} & R. Cumplimiento \\
\hline & & & & & & & & & R. Tecnología \\
\hline $\begin{array}{l}\text { Responsabilidad de } \\
\text { la dirección }\end{array}$ & 5 & & & & & & $\begin{array}{l}\text { Grupo de } \\
\text { estándares de } \\
\text { direccionamiento }\end{array}$ & 7.2 & R. Estratégico \\
\hline Talento humano & 6.2 & $\begin{array}{l}\text { Control } \\
\text { Estratégico }\end{array}$ & $\begin{array}{l}\text { Ambiente de } \\
\text { control }\end{array}$ & $\begin{array}{l}\text { Desarrollo } \\
\text { del talento } \\
\text { humano }\end{array}$ & & & $\begin{array}{l}\text { Grupo de } \\
\text { estándares de } \\
\text { gerencia del } \\
\text { Talento Humano }\end{array}$ & 7.4 & R. Operativo \\
\hline \multirow{4}{*}{ Infraestructura } & \multirow{4}{*}{6.3} & \multirow{4}{*}{$\begin{array}{l}\text { Control de } \\
\text { gestión }\end{array}$} & \multirow{4}{*}{ Información } & \multirow{4}{*}{$\begin{array}{l}\text { Sistemas de } \\
\text { información }\end{array}$} & & & \multirow{4}{*}{$\begin{array}{l}\text { Grupo de } \\
\text { Estándares de } \\
\text { gerencia del } \\
\text { Ambiente Físico }\end{array}$} & \multirow{4}{*}{7.5} & R. Cumplimiento \\
\hline & & & & & & & & & E. Imagen \\
\hline & & & & & & & & & R. Asistencial \\
\hline & & & & & & & & & R. Ocupacional \\
\hline \multirow{9}{*}{$\begin{array}{l}\text { Planificación de } \\
\text { la realización } \\
\text { del producto y/o } \\
\text { prestación del } \\
\text { servicio }\end{array}$} & \multirow{3}{*}{7.1} & \multirow{3}{*}{$\begin{array}{l}\text { Control } \\
\text { Estratégico }\end{array}$} & \multirow{3}{*}{$\begin{array}{l}\text { Direccionamiento } \\
\text { Estratégico }\end{array}$} & & \multirow{3}{*}{$\begin{array}{l}\text { Establecimiento } \\
\text { del contexto }\end{array}$} & \multirow{3}{*}{5.3} & \multirow{3}{*}{$\begin{array}{l}\text { Derechos de los } \\
\text { pacientes }\end{array}$} & \multirow{3}{*}{7.1 .1} & R. Cumplimiento \\
\hline & & & & & & & & & E. Imagen \\
\hline & & & & & & & & & R. Asistencial \\
\hline & & & & & & & & & R. Cumplimiento \\
\hline & & & & & & & & & E. Imagen \\
\hline & & & & & & & Seguridad del & & R. Asistencial \\
\hline & & & & & & & paciente & 1.1 .4 & R. Financiero \\
\hline & & & & & & & & & R. Asistencial \\
\hline & & & & & & & & & R. Financiero \\
\hline \multirow{2}{*}{$\begin{array}{l}\text { Control de } \\
\text { los equipos de } \\
\text { seguimiento y de } \\
\text { medición }\end{array}$} & \multirow{2}{*}{7.6} & \multirow{2}{*}{$\begin{array}{l}\text { Control de } \\
\text { gestión }\end{array}$} & \multirow{2}{*}{$\begin{array}{l}\text { Actividades de } \\
\text { control }\end{array}$} & \multirow{2}{*}{ Controles } & & & \multirow{2}{*}{$\begin{array}{l}\text { Grupo de } \\
\text { estándares de } \\
\text { gestión de la } \\
\text { Tecnología }\end{array}$} & 76 & R. Tecnología \\
\hline & & & & & & & & 1.0 & R. Financiero \\
\hline
\end{tabular}

Fuente: elaboración propia. 
De acuerdo con el marco teórico, los objetivos y las hipótesis de la investigación se construye una encuesta para recolectar datos sobre la forma cómo se gestionan los riesgos en la entidad. La encuesta se valida mediante una prueba piloto, con el fin de evaluar el constructo y la legibilidad, así como planeamiento, organización, ejecución y control en su aplicación; luego de la prueba piloto se realizan ajustes en el formulario. Se aplica a los líderes de los procesos asistenciales de la IPS que se enuncian a continuación: Gerente, Coordinador asistencial, Coordinador de Control Interno, Coordinador del Sistema de Gestión de la Calidad, Coordinador Talento Humano, Coordinador de sistemas de información. Las respuestas de las encuestas se tabularon mediante una tabla de Excel y se realizó el respectivo análisis de la información obtenida (Briones, 2002).

\section{RESULTADOS Y DISCUSIÓN}

Con base en los resultados del análisis de la matriz de correspondencia, en la información obtenida en las encuestas y en la revisión de documentos tanto internos de la IPS como de la bibliografía, se elabora una propuesta para la gestión integral del riesgo, la cual se planifica en concordancia con el ciclo de gestión de la calidad; la propuesta tiene como propósito facilitar la integración en la gestión de los riesgos asistenciales y administrativos que demandan los diversos documentos normativos que debe cumplir una IPS cuando opta por la acreditación de alta calidad.

La propuesta se aplica para su validación en la E.S.E. Salud Sogamoso, durante la cual se encuentra que:

- En el documento de respuesta al Modelo Estándar de Control Interno se evidencia que se llevan a cabo actividades en las cuales todos los líderes de proceso identifican sus riesgos; sin embargo, no hay socialización de dichos resultados, por esa razón en la propuesta se crean las columnas Acciones por área responsable.

- Si bien en la encuesta aplicada para identificar la metodología de gestión del riesgo se encontró que los riesgos asistenciales y de tecnología eran los de mayor probabilidad de ocurrencia en la E.S.E Salud Sogamoso, la revisión de la matriz de riesgos muestra que los riesgos asistenciales identificados están calificados con una probabilidad baja; con el fin de tener más claridad en este aspecto la propuesta se adiciona con las columnas Probabilidad de ocurrencia del riesgo y Evaluación del riesgo.

- De la encuesta se concluye que el trabajo en equipo y la identificación de responsables o líderes por área son aspectos necesarios para que la gestión del riesgo contribuya al mejoramiento continuo, aspectos que se tienen en cuenta para el diseño de la propuesta.

- Del análisis de convergencia entre el MECI, la NTC ISO 31000:2009, la NTCGP1000:2004 y el Manual de acreditación en salud ambulatorio y hospitalario, se determina la similitud de estas herramientas y se destacan sus elementos convergentes, con el fin de ilustrar las áreas o aspectos que se puede integrar durante la gestión y el cumplimiento de cada una de estas normas, así se contribuye a potenciar los recursos y cumplir con los objetivos de una manera más eficiente.

- La propuesta se presenta a la dirección de la E.S.E Salud Sogamoso para su revisión, lo que condujo a que se ajustara a la realidad de la IPS y a tener un mejor acercamiento a su aplicabilidad. Como resultado anexan a la matriz columnas para indicadores y otras denominadas: Interpretación del estándar, Fortalezas del estándar, Soportes de las fortalezas y Debilidades del estándar respectivamente; 
esto con el fin de ajustarse a la metodología para la Acreditación en salud.

- El diseño de la herramienta de aplicación de la gestión del riesgo, considerando los requisitos de la norma técnica NTC ISO 31000, para apoyar el cumplimiento de los estándares del Sistema único de acreditación en salud, es una propuesta que permite:

- Articular la gestión del riesgo y la gestión de la calidad en la atención en salud, aspectos que se deben cumplir en el ejercicio de la función pública, con el desarrollo de funciones gerenciales basadas en el ciclo de la calidad.

- La valoración del riesgo (identificación, análisis y evaluación) tanto de aquellos riesgos de origen administrativo como asistenciales que se pueden presentar en las organizaciones.

- Armonizar los procesos de la gestión del riesgo afines a las normas decretadas para la función pública.

- Establecer la ruta de acción para ejercer en las organizaciones la fase de preparación para la acreditación en salud.

La propuesta está elaborada bajo un formato de Excel; consta de 7 hojas centrales y 5 hojas auxiliares, cada una de ellas con elementos establecidos para el desarrollo de las reuniones de trabajo en las organizaciones.

\section{CONCLUSIONES}

La falta de integración de las diferentes áreas de la organización para la gestión del riesgo, así como su implementación enfocada hacia el cumplimiento de requisitos y no como un sistema que contribuye a cumplir los objetivos estratégicos de la organización, son algunas de las contradicciones que se encontraron durante el desarrollo de la investigación; por ello la propuesta de aplicación de la gestión del riesgo, basada en la norma técnica NTC-ISO 31000, facilita la integración de todas las áreas de la organización y además establece un enfoque hacia la acreditación en salud.

Se puso a prueba la segunda hipótesis planteada, dando como resultado que durante el proceso de atención al usuario no se identifica la totalidad de los riesgos, por ende no se adoptan mecanismos de prevención y gestión de tales riesgos, resultando afirmativa la hipótesis inicialmente descrita. De igual manera gracias a la matriz de correspondencia se logra probar la cuarta hipótesis, determinando que sí es posible articular la gestión del riesgo y las evaluaciones internas de calidad, para contribuir al cumplimiento de los estándares de calidad, del proceso de atención al cliente asistencial. Las hipótesis 1 y 3 no se lograron probar debido a que solo pueden refutarse o confirmarse cuando se aplique cabalmente la propuesta.

La Teoría de Reason afirma que el error aparece porque el proceso de atención no se ha diseñado con un enfoque sistémico; entonces, la consecuencia final derivada de una secuencia de procesos defectuosos, es que se favorece la aparición del error, es decir, de la no calidad en la prestación de los servicios de salud; siguiendo esta teoría como resultado de esta investigación se propone la aplicación de la gestión del riesgo dentro del marco de la norma técnica NTC ISO 31000, que considera a la organización como un sistema, para apoyar el cumplimiento de los estándares del proceso de atención al cliente asistencial del Sistema único de acreditación y facilitar la gestión de los riesgos tanto asistenciales como de índole administrativo, contribuyendo a su integración. 
La matriz de riesgos propuesta es una herramienta de gestión fundamental, pero solo contribuye al cumplimiento de los objetivos si ésta es desarrollada, socializada y continuamente revisada por todos los líderes de procesos de una Institución. Si la calificación o ponderación de la probabilidad y el impacto de los riesgos y la evaluación de los mismos es desarrollada por una sola área de la organización, es altamente probable que los resultados no correspondan a la realidad de la organización.

\section{REFERENCIAS}

Briones, G. (2002). Metodología en la investigación cuantitativa en las ciencias sociales. La encuesta social. Bogotá: ARFO editores e Impresores Ltda. ISBN:958-9329-14-4.

Chomalí, M. y Miranda, F. (2003). Gestión del riesgo en la atención de salud: hacia una cultura de la calidad basada en la seguridad. En: Revista Médica Clínica Las Condes Vol14 N. 4.

Departamento Administrativo de la Función Pública. (2005). Manual técnico del modelo estándar de control interno para el estado colombiano. Bogotá: el Departamento.

Departamento Administrativo de la Función Pública. (2011). Guía para la administración del riesgo. Bogotá: el Departamento. Cuarta edición.

Espinal, M., Yepes, L. y García, L. (2010). Seguridad del paciente, aspectos generales y conceptos básicos. Medellín: Universidad CES.

Franco, A. (2005). La seguridad clínica de los pacientes: entendiendo el problema (pp. 130-133). En:
Colombia Médica. Vol.36. Universidad del Valle abril -junio de 2005.

Hernández, R., Fernández, C. y Baptista, P. (2010). Metodología de la investigación (p. 601). Quinta edición. México: Mc Graw-Hill. ISBN: 978-607-15-0291-9.

Instituto Colombiano de Normas Técnicas y Certificación-ICONTEC. (2009a). Gestión del riesgo. Principios y directrices. NTC-ISO 31000:2009. Bogotá: el Instituto.

Instituto Colombiano de Normas Técnicas y Certificación. (2009b). Norma técnica de calidad en la gestión pública. NTCGP 1000:2009. Bogotá: el Instituto.

López, A. (2011). Fortalecimiento de competencias Investigativas. Taller segunda parte. [diapositivas]. 22 Diapositivas del módulo de Investigación I, Maestría en Calidad y Gestión Integral. Bogotá: Universidad Santo Tomás.

Ministerio de la Protección Social. (2011). Manual de Acreditación en Salud Ambulatorio y Hospitalario. Colombia. Bogotá: el Ministerio. Versión 3.

Puerto, D. (2011). La gestión del riesgo en salud en Colombia. Trabajo para optar al título de Maestría en Administración. Bogotá: Universidad Nacional de Colombia..

Reason, J. (2000). Human error: models and management. En: BMJ, 320 (7237).

Urrego, W. (2008). Cartilla No 2. Factores de riesgo ocupacional. Medellín: SERVICIO NACIONAL DE APRENDIZAJE Centro de Servicios a la Salud. 